\title{
A randomized study of combined botulinum toxin type $A$ and casting in the ambulant child with cerebral palsy using objective outcome measures
}

\author{
K. Desloovere ${ }^{a, e}$, G. Molenaers ${ }^{b}$, I. Jonkers ${ }^{a, e}$, J. De Cat ${ }^{a, d}$, L. De Borre ${ }^{b}$, J. Nijs ${ }^{a}$, \\ M. Eyssen ${ }^{c}$, P. Pauwels ${ }^{d}$ and P. De Cock ${ }^{c}$ \\ ${ }^{\mathrm{a}}$ Gait Analysis laboratory, University Hospital Leuven, Belgium, ${ }^{\mathrm{b}}$ Department of orthopaedic Surgery, University Hospital Leuven, Belgium, \\ ${ }^{\mathrm{c}}$ Department of Neuro paediatrics, University Hospital Leuven, Belgium, ${ }^{\mathrm{d}}$ Department of Rehabilitation, University Hospital Leuven, \\ Belgium, and ${ }^{\mathrm{e}}$ Department of Physical Therapy, Catholic University of Leuven, Belgium
}

\section{Keywords:}

botulinum toxin type A, casting, cerebral palsy, gait analysis
It is recognized that objective gait analysis is of great value in planning a multilevel botulinum toxin type A (BTX-A) treatment. After BTX-A treatment, objective outcome measures can provide new and interesting information for each individual child with cerebral palsy (CP). Moreover, by studying group results, we may evaluate our treatment hypotheses. The present prospective study attempts to document the effect of integrated multilevel BTX-A treatment on objective gait parameters and to define the optimal strategy for the combined treatment of BTX-A with casting in children with cerebral palsy. Objective three-dimensional gait analysis (3DGA) data were collected pre- and 2 months post-treatment, in two randomized patient groups: a first group of 17 children treated with lower leg casting prior to BTX-A injections, and a second group of 17 patients who received casting immediately after injections. The present study demonstrates that improved gait can be achieved after a multilevel BTX-A treatment, combined with casting, using a set of 90 gait parameters. The most pronounced improvement was seen at the ankle joint. The results in the knee, hip and pelvis imply that multilevel treatment of the child with CP should start at an early age, in order to prevent development of muscle contractures. Slightly more pronounced benefits, mainly in the proximal joints, were seen for the children who were casted after injections as compared to the children who were casted before injections.

\section{Introduction}

Objective gait analysis is now generally accepted in the pretreatment assessment of patients with cerebral palsy. It allows the specific description of the pattern of motion at each joint and the identification of the muscles that cause the pathological pattern, according to which treatment can be modified (Gage, 1991).

At the University Hospital of Pellenberg, objective observation of gait, in combination with extensive clinical examination followed by an additional examination under mask anaesthesia, provides decisive information for identification of target muscles during multilevel botulinum toxin type A (BTX-A) treatment. Hence, all children with cerebral palsy in whom BTX-A treatment is being considered are referred for comprehensive gait analysis including kinematics, kinetics and surface EMG.

Correspondence: Dr K. Desloovere, Gait Analysis Laboratory, University Hospital Leuven, UZ Pellenberg, Weligerveld 1, 3212 Pellenberg, Belgium

(tel.: + 32163380 14; fax: + 32163388 24;

e-mail: Kaat.Desloovere@uz.kuleuven.ac.be).
A follow-up gait analysis after BTX-A treatment allows for an objective evaluation of outcomes. In the post BTX-A treatment evaluation, we are interested in the individual's treatment result, and also in evaluating the treatment hypothesis.

The individual's treatment result provides new and interesting information that may be important in the further development or fine-tuning of that child's overall treatment strategy. By carefully evaluating the treatment outcome, we learn how the child develops new motor abilities in therapy, gait and normal daily life. Moreover, generating a 'new baseline' of the patient's gait pattern allows new recommendations aimed to preserve the therapeutic benefits.

The post BTX-A treatment evaluation can also be used to evaluate the present treatment hypothesis. We believe that gait analysis and clinical examination to compare changes before and after therapeutic intervention are crucial in developing an appropriate management strategy with BTX-A treatment for children with CP. Although BTX-A has gained widespread acceptance for the management of paediatric disorders, treatment protocols are continuously changing. Until 
recently, there has been a lack of controlled studies evaluating treatment outcomes in an objective way (Frossberg and Tedroff, 1997). Although a number of studies have examined the effect on clinical measures (Calderon-Gonzalez et al., 1994; Cosgrove et al., 1994; Koman et al., 1994; Boyd et al., 2000), few have utilized full 3DGA (three-dimensional gait analysis) for a large group of children with $\mathrm{CP}$ in a controlled prospective study (Corry, 1998; Sutherland et al., 1996; Boyd et al., 2000).

The need for this research was recognized within the gait laboratory of Pellenberg, resulting in the initiation of several studies to define the optimal strategy for integrated BTX-A-treatment, by identifying crucial factors that determine the outcome success. Following our early work using assessment of functional outcomes following BTX-A injections, several critical factors in achieving successful outcomes became clear, including: careful selection of target muscles (based on gait evaluation and clinical examination); optimal dose and injection techniques; appropriate pre- and postinjection care (serial casting, orthotic management and physiotherapy); optimal timing of the injections; and careful patient selection (using typologies of children with (P). The concept of such an approach and the importance of each of these aspects have been described previously (Molenaers et al., 1999a; Molenaers et al., 1999c).

The aim of the present paper was to establish the effect of a BTX-A treatment on objective gait parameters in order to evaluate the validity of the present treatment hypothesis of the integrated multilevel approach. From previous studies, it was concluded that casting before or after injections added supplemental benefits, resulting in an improved second rocker of the ankle with improved ankle kinetics (Molenaers et al., 1999a; Boyd et al., 2000). However, uncertainty still exists about the optimal sequence of the combination of BTX-A injections and serial casting. BTX-A works on spasticity while casting works mainly on muscle contractures. When injections precede casting, one might assume that casting will be better tolerated and contractures more easily corrected. However, because neuronal activity might improve uptake of the toxin (Hesse et al., 1998), immobilization after injections may be ill advised. The present prospective study was set up to define the optimal strategy for the combined treatment of BTX-A with casting. The description of the experimental findings will be preceded by an illustration of the additional information that can be collected from an individual's treatment result, highlighting the importance of the measurement of objective outcome data for each child.

\section{Relevance of objective outcome measures after BTX-A treatment for the individual}

First, gait analysis provides an objective baseline of the patient's walking performance that can be used as a reference for future gait analysis. A new treatment plan can be established after each analysis in order to preserve the observed improvements.

A comparison of the pre- and postinjection gait data can also help to define the capacity of the child to change his or her pathological gait pattern. For example, in children with an asymmetric gait pattern (typical for children with hemiplegia) this can be important in defining the consistency of the observed pelvic asymmetry. In these children, the more functional side is kept in front during walking, resulting in an external pelvic rotation on the pathological side. In order to keep the feet in the line of progression, a compensatory internal hip rotation is required on the pathological side. However, the cause of an internal hip rotation can also be an increased femoral anteversion, or a fixed or dynamic contracture of the medial hamstrings or other muscles. After a multilevel BTX-A treatment of all involved muscles (including medial hamstrings), some of these children may be able to change their walking pattern in the proximal segments, resulting in a neutral pelvis in the transverse plane, whereas other children will continue walking with a rotated pelvis and a compensatory hip rotation. Differentiating between these two groups can be crucial in estimating the contribution of static or dynamic contractures of the medial hamstrings to the internal hip rotation and can be very important for future treatment, such as multilevel surgery.

Additionally, treatment with BTX-A allows for the evaluation of the child's motor capacity once the tone of the spastic muscles is decreased. In particular, the functional use of the antagonist and restoration of the agonist/antagonist balance is important in this respect. An objective evaluation after BTX-A injections can also help to highlight other problems, especially the contribution of weakness, poor balance and inadequate trunk stability.

Moreover, postinjection gait analysis data is useful to distinguish between primary gait deviations and coping mechanisms. Differences between both gait deviations can be quite subtle; however, by definition, coping mechanisms will disappear spontaneously once the primary gait problems are resolved (Gage, 1991).

Finally, BTX-A treatment has a role as a presurgical evaluation method for children in whom the benefit of surgery (orthopaedic or neuro-surgery) is difficult to predict, or for whom the fine-tuning of the operation 
requires more fundamental information about underlying motor problems. Additionally, it allows for the evaluation of the child's response to intensive physiotherapy and orthotic management, which is typical as part of our postoperative rehabilitation programmes and therapeutic follow-up after BTX-A injections (Molenaers et al., 1999a).

\section{Methods}

During the past 4 years, all patients who were being considered for multilevel BTX-A treatment were evaluated by a full gait analysis, including kinematics, kinetics, surface EMG of seven lower extremity muscles, as well as a thorough clinical examination, prior to, as well as 2 months after treatment. Some patients were also re-evaluated 6 months after treatment. In young children ( $<4$ years) the gait analysis was limited to video recording, because of a lack of maintained concentration and the limited height (for an appropriate discrimination between different markers attached to the patient's body segments during gait analysis, markers may not be placed too close to each other; therefore a minimum patient height for gait analysis in our laboratory is one meter).

The inclusion criteria for the present study were that the children had to have a diagnosis of $\mathrm{CP}$, be ambulant without walking aids, and be willing to undergo full barefoot gait analysis before and after treatment. Thirty-four (34) children with predominantly spastictype cerebral palsy were included in the study. The maximal passive dorsiflexion range of motion (with the knee extended) could not be more than $10^{\circ}$. The gastrocnemius length was evaluated during the clinical examination by using the 'static' (R2) and the 'dynamic' (R1) range of motion (Boyd et al., 1999; Boyd et al., 2000). The overall mean $\mathrm{R} 2$ was $5.7^{\circ}$, indicating a mild structural shortening of the gastrocnemius. The mean
$\mathrm{R} 1$ of $-15.4^{\circ}$ indicated that there was a useful window for intervention (Boyd et al., 1999).

Seventeen patients (11 with diplegia and 6 with hemiplegia, 28 treated limbs) were treated with lower leg casting prior to BTX-A injections and a second group of 17 patients (11 diplegia and 6 hemiplegia, 28 treated limbs) were casted immediately after BTX-A injections. Each child was randomly allocated (alternate randomization) to one of the two groups. The mean age of the total group of patients was 6 years +9 months, with a range of 4 years +6 months to 9 years +10 months. All patients had physiotherapy sessions before and after BTX-A injections and comparable orthotic management involving day orthoses (AFO) and night splinting (KAFO with an external rotation/abduction rod if indicated). The medical history showed previous serial casting periods in 12 patients, whereas 16 patients had previously received multilevel BTX-A treatment. Three of the older children had an Achilles tendon lengthening at a younger age. However, no subjects received serial casting, BTX-A injections or surgery in the year previous to the treatment of the present study. Specifications of the patient cohort are given in Table 1 .

The BTX-A treatment was applied according to an integrated multilevel approach as described by Molenaers et al. (Molenaers et al., 1999b). Treatment with BTX-A was suggested due to the lack of further progression in physiotherapy, intolerance of day and night splinting, the development of fixed muscle contractures and/or a decrease in functionality. Treatment goals were defined based on objective measurements of the pathological gait and clinical examination by a multidisciplinary team that included an orthopaedic surgeon, a neuro-paediatrician, a physician, the private physiotherapist and a kinesiologist in the gait laboratory. Each selected muscle was injected at multiple sites, to a maximum of $50 \mathrm{U}$ per site, while the child was sedated with mask anaesthesia. A dilution of

Table 1 Demographics, orthotic and physiotherapy management

\begin{tabular}{lll}
\hline & Group casting post-BTX-A & Group casting pre-BTX-A \\
\hline$n$ & 17 patients, 28 treated limbs & 17 patients, 28 treated limbs \\
Diagnosis & 11 with diplegia, 6 with hemiplegia & 11 with diplegia, 6 with hemiplegia \\
Age (mean) & 7 years 0 months & 6 years 7 months \\
Day orthoses pretreatment & 6 patients & 9 patients \\
Night orthoses pretreatment & 5 patients (limited use for 4 patients) & 5 patients (limited use for 5 patients) \\
Day orthoses post-treatment & 15 patients with leafspring AFOs & 15 patients with leafspring AFOs \\
& 2 patients with hinged AFOs & 2 patients with hinged AFOs \\
Night orthoses posttreatment & 17 patients (limited use for 5 patients) & 13 patients (limited use for 4 patients) \\
Physiotherapy pretreatment & Mean of 2 sessions of $30-60$ min a week & Mean of 2 sessions of $30-60$ min a week 3 sessions of $30-60$ min a week \\
Physiotherapy post-treatment & Mean of 3 sessions of $30-60$ min a week & 6 patients \\
Previous serial casting & 6 patients & 8 patients \\
Previous BTX-A treatments & 8 patients & 2 patients \\
Previous surgery & 1 patients & \\
\hline
\end{tabular}


$100 \mathrm{U}$ of BOTOX ${ }^{\circledR}$ (Allergan Inc., Irvine, CA, USA) in $2 \mathrm{ml}$ saline was used. The total dose (calculated according to the number of selected muscles and total body weight) averaged $24.4 \mathrm{U} / \mathrm{kg}$ BW (range of 18-31 U/kg BW) for the children with diplegia and $16.4 \mathrm{U} / \mathrm{kg}$ BW (range of $12-23.5 \mathrm{U} / \mathrm{kg} \mathrm{BW}$ ) for the children with hemiplegia. The BTX-A injections were fine-tuned on a patient-by-patient basis following objective evaluation (gait analysis and clinical examination). For each treated limb between two and six muscles in at least one limb were injected in one session. All patients received injections in the gastrocnemius and medial hamstrings; other muscles injected were soleus, tibialis posterior, adductors, iliopsoas and tensor facia latae. Specifications of the doses per muscle and the different combinations of multilevel BTX-A treatments are given in Tables 2 and 3.

For all children, serial casting of the lower legs was applied over a period of 10-28 days (mean period of 20.3 days for the group who received casts before injections and mean period of 18.5 days for the group who received casts after injections). Serial stretching casts (Delta Cast Conformable ${ }^{\circledR}$ or Delta Cast Elite ${ }^{\circledR}$, Johnson \& Johnson Inc., Raynham, MA) of both lower limbs were applied with the ankle joint in neutral position or in $5^{\circ}$ of dorsiflexion (according to the individual gait pattern and muscle contractures), with the subtalar joint and midtarsal joints in a neutral position. Special care was taken to ensure that the medial arch was moulded. The casts were always removed after at least 2 weeks, and new casts were applied until an appropriate triceps surae length was achieved (with a minimum range of motion for dorsiflexion of $10^{\circ}$, depending on the baseline condition). Bilateral casting was applied for both the children with diplegia and the children with hemiplegia, in order to aim for a more symmetric gait pattern after treatment.

All patients in the present study were evaluated clinically prior to, and 4,8 and 24 weeks after treatment. A full gait analysis was performed prior to, and 2 and 6 months after treatment on each child. Additionally, treatment outcome satisfaction was evaluated using a standardized questionnaire completed by the parents and the physiotherapist.

Included in the gait analysis were three-dimensional kinematics and kinetics using a six camera VICON data capturing system and two AMTI force plates (Advanced Mechanical Technology, Inc., Watertown, MA, USA) combined with surface EMG of seven lower extremity muscles on both limbs (16 channels K-Laboratory EMG system, the Netherlands). EMG data were collected for the medial and lateral

\begin{tabular}{|c|c|c|c|c|c|c|c|c|}
\hline \multirow[b]{2}{*}{ Injected muscle } & \multicolumn{8}{|c|}{ Dose $(\mathrm{U} / \mathrm{kg})$} \\
\hline & 1 & 2 & 3 & 4 & 5 & 6 & 7 & 8 \\
\hline Gastrocnemius & 0 & 0 & 1 & 2 & 0 & 16 & 27 & 10 \\
\hline Medial hamstrings & 0 & 1 & 1 & 22 & 25 & 5 & 2 & 0 \\
\hline Lateral hamstrings & 1 & 0 & 0 & 0 & 0 & 0 & 0 & 0 \\
\hline Adductors & 10 & 20 & 2 & 0 & 0 & 0 & 0 & 0 \\
\hline Iliopsoas & 9 & 14 & 1 & 0 & 0 & 0 & 0 & 0 \\
\hline Tibialis posterior & 0 & 7 & 3 & 0 & 0 & 0 & 0 & 0 \\
\hline Soleus & 0 & 6 & 1 & 0 & 0 & 0 & 0 & 0 \\
\hline Tensor facia latae & 1 & 0 & 0 & 0 & 0 & 0 & 0 & 0 \\
\hline
\end{tabular}

Table 2 Distribution of BOTOX ${ }^{\circledR}$ doses administered in each muscle (171 injected muscles for 56 treated limbs)

\begin{tabular}{lr}
\hline Muscles injected with BTX-A in one session & Patients \\
\hline Gastrocnemius, medial hamstrings & 10 \\
Gastrocnemius, medial hamstrings, adductors & 12 \\
Gastrocnemius, medial hamstrings, iliopsoas & 9 \\
Gastrocnemius, medial hamstrings, adductors, tibialis posterior & 3 \\
Gastrocnemius, medial hamstrings, tibialis posterior & 1 \\
Gastrocnemius, medial hamstrings, leteral hamstrings, tibialis posterior & 1 \\
Gastrocnemius, medial hamstrings, adductors, iliopsoas & 10 \\
Gastrocnemius, medial hamstrings, soleus, adductors, iliopsoas & 1 \\
Gastrocnemius, medial hamstrings, soleus, adductors, tibialis posterior & 2 \\
Gastrocnemius, medial hamstrings, iliopsoas, tibialis posterior & 1 \\
Gastrocnemius, medial hamstrings, tensor facia lata & 2 \\
Gastrocnemius, medial hamstrings, adductors, soleus & 2 \\
Gastrocnemius, medial hamstrings, adductors, soleus, iliopsoas & 1 \\
Gastrocnemius, medial hamstrings, adductors, soleus, iliopsoas, tibialis posterior & 1 \\
\hline
\end{tabular}

Table 3 Distribution of the combination of target muscles injected with BTX-A during one session (56 treated limbs) 
hamstrings, rectus femoris, vastus lateralis, tibialis anterior, gastrocnemius and soleus. Vicon Clinical Manager (VCM) software (Oxford Metrics, Oxford, UK) was used to define the gait cycles and estimate the joint angles, and internal joint moments and power. The EMG signal was high pass filtered with cut-off frequency at $20 \mathrm{~Hz}(18 \mathrm{db} /$ oct, Butterworth implementation) and amplified under user control of gain, resulting in the 'raw' EMG signal. This raw signal of the EMG data was rectified and subsequent low-pass filtered (cutoff frequency at $25 \mathrm{~Hz}$ ), resulting in the linear envelope.

In order to characterize the response profiles to BTX-A treatment, all outcomes were assessed blind by an objective re-evaluation of the gait and clinical performance of each subject by the same kinesiologist. For this study, a set of 90 parameters was selected from the kinematic (joint angles), the kinetic (internal moments and power) and the EMG (linear envelope) results, as well as selective parameters derived from normal video analysis for those aspects of the gait which could not be determined from the objective kinematic data (such as foot contact, heel rise, trunk and arm motion). All selected gait parameters were defined, based on a study of the literature (Inman, 1991; Winter et al., 1987; Gage, 1991; Perry, 1992) and based on the routine gait analysis protocol used at the gait analysis laboratory of the University Hospital of Pellenberg (Catholic University of Leuven, Belgium). Data analysis included comparison of these different gait parameters, before and after treatment, within each treatment group, using a paired student $t$-test. The Wilcoxon Signed Rank test for paired variables was used to compare nonparametric variables. An unpaired student $t$-test and Wilcoxon Signed Rank was used to compare the changes across the two groups of children (casted prior or post injections). By applying a Bonferroni correction per group of parameters (kinematic, kinetic and EMG parameters) and per level (ankle, knee, hip and pelvis) an appropriate level of significance was defined as $P<0.005$.

\section{Results}

This paper focuses on the results of the objective gait analysis pre- and 2 months post treatment including kinematics, kinetics, EMG data and a set of selective parameters estimated from the normal video analysis. As not all subjects have completed the 6 months posttreatment course, a description of the repeated clinical examination, gait analysis and questionnaire at 6 months post-treatment is not reported here. Tables 4-8 show the mean parameters (with SD) pre- and postBTX-A treatment for both groups, as well as normal values, which is a mean for a group of 10 adults, for different gait parameters. Nonparametric variables and EMG (timing) parameters were not included in these tables. It was decided to compare the data with normal data of adults, because of the wide range of age in the

Table 4 Mean and $(S D)$ of ankle and foot parameters for the group of children who received casts after injections $(n=28)$ and the group of children who received casts before injections $(n=28)$, and for adult normal values $(n=10)$

\begin{tabular}{|c|c|c|c|c|c|c|c|}
\hline \multirow[b]{2}{*}{ Gait parameter } & \multicolumn{3}{|l|}{ Casting Post } & \multicolumn{3}{|l|}{ Casting Pre } & \multirow[b]{2}{*}{ Normal } \\
\hline & Pre & Post & $P$ & Pre & Post & $P$ & \\
\hline Ankle angle at initial contact $\left(^{\circ}\right)$ & $-4.1(6.1)$ & $1.82(5.1)$ & $* * * *$ & $-6.4(8.8)$ & $-0.5(7.3)$ & $* * *$ & 0 \\
\hline \multicolumn{8}{|l|}{ Maximum ankle dorsiflexion between } \\
\hline loading response and midstance $\left({ }^{\circ}\right)$ & $5.43(5.7)$ & $8.64(6.4)$ & $* * *$ & $5.4(8.2)$ & $8.2(6.4)$ & & 0 \\
\hline Range of ankle motion during push-off $\left({ }^{\circ}\right)$ & $20.1(4.2)$ & $17.9(6.7)$ & & $21.6(7.0)$ & $19.7(6.5)$ & & 25 \\
\hline $\begin{array}{l}\text { Timing of maximum ankle dorsiflexion } \\
\text { in stance }(\%)\end{array}$ & $41.4(12.0)$ & $49.2(3.6)$ & $* * *$ & $46.3(7.0)$ & $50.1(6.5)$ & & 50 \\
\hline Maximum ankle dorsiflexion in stance $\left(^{\circ}\right)$ & $4.1(6.1)$ & $11.5(6.0)$ & $* * * *$ & $4.57(8.4)$ & $10.8(5.6)$ & $* * *$ & 10 \\
\hline Ankle angle at mid-swing $\left({ }^{\circ}\right)$ & $-7.5(7.1)$ & $0.82(7.0)$ & $* * * *$ & $-10.4(9.3)$ & $-0.8(6.1)$ & $* * * *$ & -2 \\
\hline Range of ankle motion in swing $\left(^{\circ}\right)$ & $15.6(6.3)$ & $11.9(4.5)$ & $* * *$ & $16.1(7.0)$ & $11.4(4.6)$ & $* * * *$ & 18 \\
\hline Mean foot alignment in stance $\left(^{\circ}\right)$ & $3.25(11.5)$ & $-3.82(10.7)$ & $* * * *$ & $-10.7(12.0)$ & $-5.1(14.4)$ & & -4 \\
\hline Mean foot rotation in stance $\left(^{\circ}\right)$ & $-7.7(9.7)$ & $-12.7(8.2)$ & $* * *$ & $-4.3(12.4)$ & $-6.8(10.4)$ & & -13 \\
\hline Ankle moment at loading response $(\mathrm{Nm} / \mathrm{kg})$ & $0.85(0.36)$ & $0.54(0.28)$ & $* * * *$ & $0.88(0.4)$ & $0.5(0.3)$ & $* * * *$ & 0 \\
\hline $\begin{array}{l}\text { Maximum plantar flexion moment in mid- } \\
\text { and terminal stance }(\mathrm{Nm} / \mathrm{kg})\end{array}$ & $1.04(0.26)$ & $0.77(0.22)$ & $* * *$ & $1.14(0.3)$ & $0.77(0.2)$ & $* * * *$ & \\
\hline $\begin{array}{l}\text { Maximum plantar flexion moment at preswing } \\
(\mathrm{Nm} / \mathrm{kg})\end{array}$ & $0.88(0.19)$ & $0.99(0.2)$ & $* * *$ & $0.83(0.2)$ & $0.97(0.1)$ & $* * *$ & 1.5 \\
\hline $\begin{array}{l}\text { Ankle power absorption at loading response } \\
(\mathrm{W} / \mathrm{kg})\end{array}$ & $-1.42(0.5)$ & $-0.75(0.6)$ & $* * * *$ & $-1.36(0.7)$ & $-0.84(0.6)$ & $* *$ & 0 \\
\hline Ankle power generation at preswing $(\mathrm{W} / \mathrm{kg})$ & $1.22(0.47)$ & $1.33(0.57)$ & & $1.13(0.7)$ & $1.19(0.5)$ & & 3 \\
\hline
\end{tabular}

${ }^{*} P<0.05,{ }^{* *} P<0.01,{ }^{* * *} P<0.005, * * * * P<0.001$. 
Table 5 Mean and $(S D)$ of knee kinematic parameters for the group of children who received casts after injections $(n=28)$ and the group of children who received casts before injections $(n=28)$, and for adult normal values $(n=10)$

\begin{tabular}{|c|c|c|c|c|c|c|c|}
\hline \multirow[b]{2}{*}{ Gait parameter } & \multicolumn{3}{|l|}{ Casting Post } & \multicolumn{3}{|l|}{ Casting Pre } & \multirow[b]{2}{*}{ Normal } \\
\hline & Pre & Post & $P$ & Pre & Post & $P$ & \\
\hline Knee angle at initial contact $\left(^{\circ}\right)$ & $26.6(11.2)$ & $25.1(10.9)$ & & $24.1(11.1)$ & $20.9(12.5)$ & & 7 \\
\hline Maximum knee flexion in stance $\left({ }^{\circ}\right)$ & $28.1(10.1)$ & $27.6(10.5)$ & & $25(11.7)$ & $23(13.9)$ & & 19 \\
\hline Timing of knee extension motion ( $\%$ of gait cycle) & $6.11(5.9)$ & $8.2(5.1)$ & $*$ & $6.6(5.5)$ & $7.3(5.2)$ & & 17 \\
\hline Knee angle at toe off $\left({ }^{\circ}\right)$ & $30.0(8.8)$ & $29.9(10.6)$ & & $27.3(8.8)$ & $26.4(7.1)$ & & 40 \\
\hline Maximum knee extension in stance $\left(^{\circ}\right)$ & $7.61(9.6)$ & $4.53(7.7)$ & $*$ & $-0.04(10.2)$ & $-0.71(7.3)$ & & 0 \\
\hline Range of knee motion in sagittal plane in stance $\left(^{\circ}\right)$ & $21.9(7.1)$ & $26.3(9.8)$ & $*$ & $27.9(9.4)$ & $29.5(9.0)$ & & 35 \\
\hline Maximum knee flexion in swing $\left({ }^{\circ}\right)$ & $58.6(6.4)$ & $57.4(7.2)$ & & $54.7(9.0)$ & $52.6(10.0)$ & & 57 \\
\hline Amount of delayed knee flexion in swing $(\%)$ & $18.6(4.5)$ & $16.1(3.6)$ & $* * *$ & $16.1(7.2)$ & $15.4(3.3)$ & & 12 \\
\hline
\end{tabular}

$* P<0.05, * * P<0.01, * * * P<0.005, * * * P<0.001$.

Table 6 Mean and $(S D)$ of hip kinematic parameters for the group of children who received casts after injections $(n=28)$ and the group of children who received casts before injections $(n=28)$, and for adult normal values $(n=10)$

\begin{tabular}{|c|c|c|c|c|c|c|c|}
\hline \multirow[b]{2}{*}{ Gait parameter } & \multicolumn{3}{|l|}{ Casting Post } & \multicolumn{3}{|l|}{ Casting Pre } & \multirow[b]{2}{*}{ Normal } \\
\hline & Pre & Post & $P$ & Pre & Post & $P$ & \\
\hline Hip flexion at initial contact $\left({ }^{\circ}\right)$ & $42.8(5.0)$ & $43.7(8.2)$ & & $42.8(10.0)$ & $42.5(10.0)$ & & 38 \\
\hline Hip angle at terminal stance $\left({ }^{\circ}\right)$ & $-3.3(5.8)$ & $-2.6(5.5)$ & & $-5(7.0)$ & $-5.4(8.9)$ & & -10 \\
\hline Range of Sagittal hip motion in stance $\left(^{\circ}\right)$ & $46.1(6.3)$ & $46.8(9.4)$ & & $48.8(7.5)$ & $47.8(12)$ & & 48 \\
\hline Maximum hip flexion in swing $\left({ }^{\circ}\right)$ & $46.8(5.0)$ & $47.5(7.2)$ & & $47.4(7.6)$ & $46.3(8.2)$ & & 37 \\
\hline Range of hip ab- and adduction $\left({ }^{\circ}\right)$ & $17.5(3.7)$ & $16.9(5.1)$ & & $17.6(3.7)$ & $16.8(4.0)$ & & 15 \\
\hline Mean coronal hip angle in stance $\left(^{\circ}\right)$ & $3.4(5.0)$ & $2.1(5.6)$ & & $2.42(5.0)$ & $2(5.4)$ & & 3 \\
\hline Mean coronal hip angle in swing $\left({ }^{\circ}\right)$ & $-3.7(5.2)$ & $-3.9(4.7)$ & & $-3.9(4.4)$ & $-4.2(4.3)$ & & -3 \\
\hline Hip rotation angle at initial contact $\left(^{\circ}\right)$ & $5.43(9.7)$ & $2.78(9.6)$ & & $-0.93(10)$ & $-2.1(9.9)$ & & -7 \\
\hline Hip rotation angle at midstance $\left({ }^{\circ}\right)$ & $13.6(10.1)$ & $11.3(9.1)$ & & $4.93(10)$ & $8.53(11)$ & & 5 \\
\hline Hip rotation angle at terminal stance $\left(^{\circ}\right)$ & $13.7(9.9)$ & $11.7(9.9)$ & & $3.32(10.1)$ & $6.9(8.8)$ & & 7 \\
\hline Hip rotation angle at midswing $\left({ }^{\circ}\right)$ & $11.7(8.7)$ & $9.6(7.4)$ & & $5.3(10.7)$ & $3.9(8.5)$ & & 6 \\
\hline
\end{tabular}

$* P<0.05, * * P<0.01, * * * P<0.005, * * * * P<0.001$.

Table 7 Mean and $(S D)$ of pelvic parameters for the group of children who received casts after injections $(n=28)$ and the group of children who received casts before injections $(n=28)$, and for adult normal values $(n=10)$

\begin{tabular}{|c|c|c|c|c|c|c|c|}
\hline \multirow[b]{2}{*}{ Gait parameter } & \multicolumn{3}{|l|}{ Casting Post } & \multicolumn{3}{|l|}{ Casting Pre } & \multirow[b]{2}{*}{ Norma } \\
\hline & Pre & Post & $P$ & Pre & Post & $P$ & \\
\hline Pelvis mean anteversion $\left({ }^{\circ}\right)$ & $18.7(3.9)$ & $20.0(4.5)$ & & $21.7(5.6)$ & $21.4(4.8)$ & & 14 \\
\hline Range of pelvic motion in sagittal plane $\left(^{\circ}\right)$ & $9.1(2.6)$ & $8.8(2.3)$ & & $7.8(2.5)$ & $7.9(2.8)$ & & 1 \\
\hline Range of pelvic obliquity $\left({ }^{\circ}\right)$ & $12.5(3.6)$ & $12.1(5.3)$ & & $13.6(3.9)$ & $13.5(3.8)$ & & 12 \\
\hline Mean pelvic obliquity $\left({ }^{\circ}\right)$ & $2.17(7.7)$ & $1.06(5.7)$ & & $2.42(6.0)$ & $1.28(6.0)$ & & 0 \\
\hline Range of pelvic rotation $\left(^{\circ}\right)$ & $20.2(5.1)$ & $17.7(4.5)$ & $*$ & $19.9(6.3)$ & $19.0(7.4)$ & & 6 \\
\hline Mean pelvic rotation $\left({ }^{\circ}\right)$ & $5.0(10)$ & $3.7(11.9)$ & & $1.83(12)$ & $0.28(8.9)$ & & 0 \\
\hline
\end{tabular}

$* P<0.05, * * P<0.01, * * * P<0.005, * * * * P<0.001$.

patient cohort (4 years 6 months to 9 years 10 months). The normal database for children collected in the gait laboratory of Pellenberg showed that mean values of a group of children with an age range of 3-6 years does not significantly differ from the mean values of adults; only the variation within and between subjects was found to be significantly larger for children as compared to adults (Desloovere et al., 1998).

\section{Time and distance parameters}

No significant between group differences were reported pre- and post-treatment for the time and distance parameters. Following treatment, walking velocity of both groups decreased slightly, with a mean decrease of $0.01 \mathrm{~m} / \mathrm{s}$ for the group receiving casts after injections and $0.05 \mathrm{~m} / \mathrm{s}$ for the group receiving casts before injections. 
Table 8 Maximal linear envelope of EMG (dynamic rectified EMG recordings in minivolts) for the group of children who received casts after injections $(n=28)$ and the group of children who received casts before injections $(n=28)$

\begin{tabular}{lllllllll}
\hline & \multicolumn{3}{c}{ Casting Post BTX-A } & & \multicolumn{3}{c}{ Casting Pre BTX-A } \\
\cline { 2 - 3 } Muscle group & Pre & Post & $P$ & & Pre & Post & $P$ \\
\hline Max. activity of medial hamstrings & 238.2 & 160.3 & $* * * *$ & & 219 & 137 & $* * * *$ \\
Max. activity of lateral hamstrings & 191 & 176 & 0.29 & & 214 & 164 & $* * * *$ \\
Max. activity of rectus femoris & 192 & 183 & 0.44 & & 182 & 147 & $*$ \\
Max. activity of vastus lateralis & 238 & 231 & 0.74 & & 250 & 221 & 0.13 \\
Max. activity of tibialis anterior & 160 & 143 & 0.067 & & 155 & 119 & $* * * *$ \\
Max. activity of gastrocnemius & 226 & 142 & $* * * *$ & & 262 & 161 & $* * * *$ \\
Max. activity of soleus & 172 & 142 & $*$ & & 225 & 167 & $* * * *$ \\
\hline
\end{tabular}

$* P<0.05, * * P<0.01, * * * P<0.005, * * * * P<0.001$

\section{Foot and ankle parameters}

For both groups, all ankle kinematic parameters (Table 4) improved significantly 2 months after treatment $(P<0.005)$, except for the ankle range of motion during push off and the maximal dorsiflexion angle between loading response and midstance. The latter parameter showed a trend toward increased dorsiflexion. The mean change in initial contact dorsiflexion was almost $6^{\circ}$, resulting in a slight over-correction. However, the mean improvement in stance dorsiflexion was $6.8^{\circ}$, indicating an almost complete normalization. The beneficial results of ankle motion in stance was confirmed by two other (nonparametric) variables, estimated from the analysis of the normal video frames: For the total group, toe-contact was noticed in $41 \%$ of the treated limbs before treatment, and only in $6 \%$ after treatment. Before injections, $62 \%$ of the treated limbs showed a premature heel rise in stance, decreasing to $27 \% 2$ months after injections. Additionally, statistically significant improvement in swing phase dorsiflexion $(P<0.005)$ and range of ankle motion in swing was demonstrated.

The double bump pattern of the ankle moment (estimated from a nonparametric and from a parametric variable) as well as power absorption during loading response decreased significantly (Table 4$)$. The maximal ankle plantar flexion moment at the end of stance increased significantly $(P<0.005)$, although this was not sufficient enough to increase power generation at push off to reach statistical significance.

For the group receiving casts after injections, the feet were significantly more externally aligned during stance 2 months after treatment as compared with the baseline alignment.

\section{Knee parameters}

With a critical $P$-value at 0.005 , no knee parameters (Table 5) were found to have changed significantly between 3 months postinjection and baseline condition. Although the knee angle at initial contact improved in both groups, statistical significance was not achieved. The group of children who received casts after injections demonstrated a trend towards improved knee extension and increased range of knee motion in stance $(P<0.05)$, as well as a significant decrease of the delayed knee flexion during swing $(P<0.005)$. Additionally, based on video observation, a significant decrease in the frequency of pathological plantarflexion/knee-extension couple was demonstrated: from $61 \%$ of the treated limbs at baseline, to $27 \%$ of the treated limbs 2 months after treatment (total group results).

\section{Hip and pelvis}

Almost no hip and pelvic parameters were found to be significantly different between baseline and 2 months postinjection (Tables 6 and 7). Both groups showed a slight trend towards a decrease of internal hip rotation at initial contact (with about $3^{\circ}$ of change), although this did not reach statistical significance. The group with casting before injections showed a significant decrease in hip power generation in stance (not included in Table 6). Additionally, both groups showed a tendency towards decreased range of pelvic motion in all planes.

\section{Muscle activity parameters}

Table 8 shows maximal values of the semiquantitative analysis of dynamic rectified EMG recordings (linear envelope), for seven muscle groups. Comparing the linear envelope across session is problematic from an EMG quantification standpoint. Nevertheless, both groups showed a systematic and significant decrease of maximal muscle activity of the medial hamstrings and gastrocnemius muscle during gait 2 months after injections as compared with their baseline condition. For the group with casting before treatment, a decrease of activity was also noticed for the lateral hamstrings (not injected in the group of children who received casts before injections and in one patient in the group of 
children who received casts after injections) and for the soleus muscle (injected in four patients for the group of children who received casts before injections and in two patients in the group of children who received casts after injections). All other muscle groups showed a decrease in activity, although statistical significance was not reached.

\section{Side-effects}

Although side-effects are not the main issue of this paper, it is important to note that no important adverse effects of the combined BTX-A and casting treatment were noted for the doses applied. Minor complications were seen in 14 children, equally distributed over both groups. Twelve children showed generalized weakness during 1-2 weeks, while temporary incontinence was reported three times and constipation was reported once. Weakness was not seen as a major complication, but more as an expected side-effect due to the decreased spasticity of the agonist muscle, muscle atrophy that occurred during the casting period and the time that the child required to shift agonist/antagonist balance. These factors were deemed most likely to contribute to the problems of weakness and poor posture control initially observed following treatment.

\section{Discussion}

\section{General changes in gait parameters after multilevel BTX-A injections combined with casting}

Time and distance parameters

Although only a small decrease in mean walking velocity was reported for both groups, the functional improvements 2 months after treatment seemed to vary from subject to subject. Fifty per cent of the children actually showed an increased walking velocity after treatment, due to an increased step length and an increased cadence. Preliminary results of the gait evaluation 6 months after treatment (results not included in this paper) revealed significantly increased walking velocity and step length compared with baseline values.

The initial decrease of mean walking velocity is probably due to the impact of potential underlying weakness, once the spasticity is partly removed and the weakness caused by the injections and by casting (muscle atrophy). The antagonists need time to improve in strength and functional control during the period of denervation of the spastic muscles. Moreover, some children who received casting for more than 2 weeks after injections, might still feel slightly uncertain at the time of the post-treatment gait analysis and could be weak. The mean period of casting for the children that were casted after injection was 18.5 days (with a range of 10-28 days) with the first post-treatment gait analysis planned 8 weeks after injections. Previous studies (Sutherland et al., 1996; Motta, 1997; 1999; Wissel et al., 1999) showed a significantly increased walking velocity and step length 3-12 months after BTX-A treatment; however, these studies only included gastrocnemius muscle injections.

\section{Foot and ankle parameters}

With respect to changes in the ankle parameters in both groups (Table 4), significantly improved ankle position at initial contact, during second rocker and during swing were observed $(P<0.005)$. Treatment targeted toward reducing the dominance of the ankle-plantar flexors over the antagonist muscles resulted in an increased ankle dorsiflexion in both stance and swing. This favourable effect after injecting the calf muscle was also seen in other studies (Cosgrove et al., 1994; Sutherland et al., 1996; Boyd and Graham, 1997; Motta, 1997; Corry et al., 1998; Boyd et al., 1999; Molenaers et al., 1999a; Wissel et al., 1999; Boyd et al., 2000).

The improvements in ankle kinematics had a beneficial impact on the ankle kinetics. Apart from a decrease in ankle moment double bump pattern as well as an improvement in ankle power absorption, a significant increase of ankle plantar moment at push off was observed. Such an increase in plantar flexion moment should contribute to an increase of power generation at push off. In fact, this favourable effect did occur, however, this latter change did not reach statistical significance at 2 months post-treatment. A decreased walking velocity (resulting in a decreased angular velocity) may be the cause for the limited change in ankle power generation. With an increased walking velocity at 6 months postinjection (results not included in this paper) the ankle power generation at push off is expected to be more pronounced. Similar improvements in ankle kinetics were described by Boyd et al. (2000) in children with cerebral palsy. They described that the pathological 'double bump' ankle moment and the abnormal 'triphasic' ankle power curve improved significantly after BTX-A injection in the gastroc-soleus muscle.

The results of the group receiving casts after injection revealed a significant decrease of the internal foot alignment in stance. This correction could be attributed to a decrease in pelvis, hip and foot rotation. The improvements in foot rotation (alignment of the foot with regards to the tibia) involved a decrease of varus position, due to injection of the tibialis posterior (applied in seven of 17 patients in the group receiving 
casts after injections, compared with one of 17 patients in the group receiving casts before injections). The group receiving casts before injections did not show such a pathological foot alignment at baseline, indicating that the tibialis posterior was not a target muscle for injection.

\section{Knee, hip and pelvis parameters}

Limited objective data is available on the changes in the proximal joints after multilevel BTX-A treatment (Frossberg and Tedroff, 1997). Cosgrove et al. (1994) and Corry et al. (1999) reported improved knee kinematics after injecting the hamstrings. Sutherland et al. (1996) reported that the knee angle at initial contact did not change; however, this was after an isolated injection into the gastrocnemius muscle. None of these studies reported the effect of a multilevel BTX-A treatment on the hip and pelvic motion.

The present study reports a decrease of the pathological plantar-flexion/knee-extension couple 2 months after treatment for both groups. This was defined based on the parameters derived from the video, but was also verified by an increase of the maximal dorsiflexion ankle in stance and the timing of the start of push-off (Table 4), and by a delayed start of the knee extension motion in stance (Table 5). Although a slight decrease of knee flexion at initial contact was seen in $50 \%$ of the cohort, mean changes were minimal and were not found to be significant. Knee kinetic results (not included in Table 5) revealed a trend towards an increased knee extension moment in stance. This was not due to an increase of knee flexion in stance (knee angle in stance actually decreased), but should be related to the improved ankle kinematics (first and second rocker), preventing an abnormally early forward progression of the ground reaction force.

Since all patients received injections in the medial hamstrings, a decrease in internal hip rotation at initial contact was seen in $50 \%$ of the treated limbs, coinciding with the instant of maximal stretch on the hamstrings. The positive trend in changes in knee flexion in stance could be explained accordingly; however, these differences did not reach statistical significance. It should also be noted that other muscles than the medial hamstrings might have a large contribution to internal hip rotation (Delp et al., 1996). The results of the hip kinetics (not included in Table 6) revealed that a hip flexion moment occurred earlier in stance phase 2 months after treatment as compared with baseline. The reduction of prolonged activity of the medial and lateral hamstrings in stance could be related to this observation.

Pelvic asymmetry in frontal and transverse plane decreased in $40 \%$ of the children. The capacity to change pelvic asymmetry after an individually tuned multilevel BTX-A treatment can be important information for the course of future treatment. Although the range of motion of the pelvis decreased in the three motion planes after BTX-A injections, the most pronounced results were found in the transverse plane for the group receiving casts after injections $(P=0.013)$. This seems to confirm the improved pelvic stability after BTX-A treatment, reported in previous studies (Molenaers, 1999a, 1999b). The flat foot position in stance 2 months after treatment could explain the improved stability in the proximal joints, as compared to the pretreatment condition. Improved stability in the distal joints reduces the need for fixation in the proximal joints, resulting in a decrease of pelvic rotation.

\section{Muscle activity parameters}

No significant improvement in phasic activity of the medial hamstrings, gastrocnemius and soleus (frequently injected muscles) was noticed at 2-month follow-up. There was no reason to expect changes in phasic activity of these muscles, which remained under abnormal central nervous system control after partial paresis. Table 8 illustrates that the injected muscles still showed a significantly decreased activity 2 months after treatment. This was most pronounced for the medial hamstrings and gastrocnemius muscles (muscles that were always injected). The lateral hamstrings and soleus were less frequently injected in the patient cohort. The lateral hamstrings were only injected once with $1 \mathrm{U}$ BOTOX ${ }^{\circledR}$ per $\mathrm{kg}$ body weight, and the soleus muscle was injected in six of the 12 children with hemiplegia, mainly in the group receiving casts before injections.

Based on analysis of the maximal activity of the soleus muscle for a subgroup of the patient cohort for whom no soleus injections were applied, we found that the activity of the soleus muscle did not change significantly after treatment, indicating that there is no evidence for overflow of BTX-A from the gastrocnemius muscle into the soleus. This was also seen in previous studies (Molenaers, 1999b). Semi-quantitative analysis of the EMG data for the noninjected tibialis anterior muscle also revealed that a multilevel injection procedure produces a reduction of activity. A decrease in tibialis anterior activity during stance was seen previously by Sutherland et al. (1996).

\section{Overall results}

Overall examination of the results clearly demonstrates that multilevel BTX-A treatment combined with casting results in significant changes to the walking pattern. Although a multilevel treatment was applied for all patients, the most significant changes were seen at the ankle joint, with less pronounced changes at the knee, hip and pelvis. The application of lower leg casts that 
only cross the ankle joint could contribute to the more expressed changes observed at that joint. Additionally, the patient distribution of 22 children with diplegia vs. 12 children with hemiplegia could contribute to the limited changes observed at the more proximal joints. Children with diplegia are more likely to present with greater involvement of the proximal muscles (such as medial hamstrings and hip flexors). These children are therefore more likely to develop fixed contractures at the level of the hamstrings and hip flexors, as compared with children with hemiplegia who are more likely to develop fixed contractures of the gastroc-soleus muscle. Since fixed contractures are not sensitive to BTX-A, these factors may have contributed to the observed treatment outcomes.

Additionally, the age of the children within the study population (ranging from 4 years 3 months to 9 years 10 months) could have contributed to the limited changes observed at the proximal joints. The inclusion criterion that patients had to undergo a full gait analysis artificially increased the mean age for the BTX-A group in this study (for children younger than 4 years old the gait analysis is limited to video recording). At an older age muscle contractures are more common, as could be determined from the results of the clinical examinations that indicated mild medial hamstring contractures before treatment. Based on the clinical exam, contractures were shown to decrease significantly in both groups 4 weeks and 2 months after treatment, although these decreases were not sufficient enough to significantly change the walking pattern. The more discrete gain seen in the proximal joints due to the presence of contractures provides evidence that early treatment at all involved levels is necessary to prevent contractures. Since BTX-A is most effective in patients with dynamic contractures, repeated multilevel BTX-A treatment can help to prevent the development of contractures in the proximal joints, if started at an early age. Furthermore, BTX-A is more likely to demonstrate a disease modifying effect when administered during the period of motor development (Boyd and Graham, 1997; Graham et al., 2000).

The multilevel administration of BTX-A follows the rationale established for pure orthopaedic interventions, based on the work of Wenger and Rang (1993). They presented evidence that a single surgical procedure to lengthen and transfer the involved muscles is more successful in simultaneously balancing all lowerextremity joints. The same principles as those used for multilevel surgery should be applied in the selection of target muscles for BTX-A injections, with treatment focused on the optimal alignment of the lower legs and pelvis, allowing the child to develop and train a new walking pattern. When examining the results in detail, a specific subgroup of children with $\mathrm{CP}$ can be identified who clearly improve their gait parameters related to the control of hip and pelvis. These so-called 'excellent' responders who improve their gait at all levels after treatment, are characterized by the absence of severe contractures in the proximal joints. These children provide further evidence that multilevel BTX-A injections are most effective if used at an early age, thereby preventing the development of muscle contractures. Accordingly, goal setting for multilevel BTX-A treatment in older children should be modified and the application of removable upper-leg casts might be considered more frequently in these children.

\section{Comparison of the two treatment groups}

When comparing the results from the group of children who received casts before injections with the group of children who received casts after injections, we found slight differences mainly in the more proximal joints (knee, hip and pelvis). The unpaired statistical analysis to compare changes across the two randomized groups revealed no significant differences; however, some issues that might be important for future treatment are described below.

Limited data are available on the effect of a combination of BTX-A injections and serial casting (Molenaers et al., 1999b; Boyd et al., 2000). However, from this study data the additional benefits of the combined treatment became clear. Corry et al. (1998) and Flett et al. (1999) compared the effect of BTX-A injections into the calf with the effect of serial casting and concluded that there was a longer lasting effect for the BTX-A group as compared to the casting group. No previous studies demonstrated the effect of the timing of the casting within a combined BTX-A/casting treatment (pre or post BTX-A injections).

There is an ongoing discussion as to the optimal combination of BTX-A injections and serial casting in the management of CP. BTX-A works on spasticity while casting mainly works on early contractures. By injecting first, it can be assumed that casting will be better tolerated and that muscle contractures will be more easily corrected. However, any neuronal activity improves uptake of the toxin (Hesse et al., 1998), suggesting that immobilization after injections is not the ideal solution. Moreover, Suliman et al. (1997) indicated that junctional and extajunctional nicotinic acetylcholinergic receptors in the neuromuscular junctions significantly increased in the soleus and tibialis anterior muscles in rats after 4 weeks of immobilization. One may expect that an increased number of acetylcholine receptors after a casting period before injections would require an increased dose of BTX-A 
in order to achieve a similar effect. The present study offers relevant observations in an attempt to define the best strategy for the combination of treatment with BTX-A and serial casting in children with spastic CP with mild muscle contractures.

The initial scores for the gait parameters were not significantly different between both groups, indicating similar pretreatment conditions, except for foot alignment, hip rotation at initial contact and minimal knee angle in stance.

Although both groups had similar pretreatment conditions (Table 1), the group of children who received casts before injections reported more frequent problems with compliance of night splints (four children did not tolerate night splints) as compared with the group who received casts after injections (all children tolerated night splints). Compliance of day and night splints has proven to be a crucial factor for a prolonged benefit of a BTX-A treatment (Molenaers, 1999a). The patients who received casts after injections needed a shorter period for correcting the triceps contractures (with mean casting period of 18.5 days), as compared to the patients who received casts before injections (with a mean casting period of 20.3 days), which may be an indication that triceps surae contractures were more easily corrected.

For the ankle and foot parameters, the group of children with casts after injections showed a more pronounced internal foot alignment at baseline. Based on this finding, the tibialis posterior was more frequently selected for injection resulting in a significantly improved foot alignment after treatment. For the group of children who received casts before injections the mean foot alignment was almost normal at baseline. Accordingly, for this group, the tibialis posterior was less frequently selected as a target muscle, and consequently no significant change of this parameter was expected. Other parameters of foot and ankle showed similar improvements in both groups. When comparing the results of the present study with the results of a previous study where no casts were applied (Molenaers et al., 1999a), more pronounced improvements were seen when applying casts. The present study therefore supports the benefit of combined treatment of BTX-A and casts as described previously (Molenaers et al., 1999b; Boyd et al., 2000; Desloovere et al., 2000).

At the knee joint, a correction of the pathological plantar flexion/knee extension couple could be inferred from the timing of the knee extension motion and the timing of maximal ankle dorsiflexion in stance for both groups (Tables 4 and 5). This effect was slightly more pronounced for the group of children who received casts after injections as compared to the children with casts before injections. Additionally, the maximal ankle dorsiflexion angle between loading response and midstance improved significantly for the group of children who received casts after injections. A similar trend, although not significant, was seen in the group with casts before injections. This over-correction may be due to poor muscle control of the denervated gastroc-soleus muscle and a loss of confidence shortly after casting. Especially in the group with casts after injection the period between the removal of the casts and the gait analysis was shorter as compared with the group of children who received casts before injections, indicating that some children of the former group (seven patients of the group received casting for more than 2 weeks) might still feel slightly uncertain at the time of the posttreatment gait analysis (8 weeks after injections).

At baseline, the group of children who received casts after injections typically showed a knee flexion pattern, whereas the group of children with casts before injections more frequently showed a knee (hyper) extension pattern in stance. This was concluded based on the results of the minimal knee angle in stance (Table 5). The multilevel BTX-A injections had no effect on the minimal knee angle in stance for the group of children who received casts before injections; however, the group of children who received casts after injections showed a decrease of the mean minimal knee angle in stance from $7.6^{\circ}$ to $4.5^{\circ}(P=0.021)$. Both findings indicate that there was no increase of crouch due to a weakened gastroc-soleus muscle. The combined injection of gastrocnemius and both medial hamstrings and psoas muscle is believed to be crucial in order to prevent crouch.

The maximal range of motion of the pelvis in the three motion planes was decreased for both patient groups, indicating improved pelvic stability. This finding was most pronounced for the transverse plane motion in the group of children with casts after injections. This seems consistent with the fact that casts, if applied bilaterally, provide stability during gait and therefore reduce the need of proximal fixation at the level of the hip and pelvis, particularly in the transverse plane. The children who received casts after injections can profit from the improved stability and the decreased muscle tone at all injected levels simultaneously. The group who received casts before injections may also profit from the improved stability during the casting period; however, at that time, the proximal joints are not yet treated. These children will have to adapt twice to new situations: a first time while walking with casts and a second time after tone reduction at different levels. The first adaptation may cause compensatory motion at the proximal joints. The physiotherapists working with the children who received casts before injections still have to manage increased tone 
in the proximal joints during the casting period. The authors believe that the children who received casts after injections profit from the simultaneous benefit of improved stability and tone reduction at all levels. Additionally, physiotherapy sessions during the casting period can focus more effectively on problems of the proximal joints, by trying to gain muscle length (in hamstrings and hip flexors) and by training pelvis and trunk control. These children can learn the specific targeted movement pattern advised by their physiotherapists when trying to achieve a new walking pattern from the beginning of the treatment and they do not have to adapt to new situations twice.

\section{Conclusion}

Results of the present study demonstrate that an integrated multilevel treatment with BTX-A combined with casting and appropriate physiotherapy and orthotic management is successful in managing spasticity in children with $\mathrm{CP}$, and results in improved walking performance. BTX-A treatment is therefore a useful adjunct to the interdisciplinary care of these children, particularly at a young age, when the gait pattern can still be adapted and the spastic muscles stretched so that contractures can be prevented. Objective gait data document that the greatest improvements are observed at the ankle joint. The results in the knee, hip and pelvis imply that multilevel treatment should start at an early age, in order to prevent development of muscle contractures. There were slightly more pronounced benefits, mainly in the proximal joints, for the children who were casted after injections. It is believed that the children may benefit from the simultaneous improved stability during casting and muscle tone reduction at all levels.

It is our belief that gait analysis is of great value for planning BTX-A treatment and documenting the outcome for ambulant children. With the additional insights provided by future research, the predictability of treatment outcomes should further be improved. The use of pattern recognition in the gait analysis data may help to identify gait characteristics of mild, good and excellent responders to BTX-A treatment, and to improve the prognosis for these patients.

\section{References}

Boyd R, Graham HK (1997). Botulinum toxin A in the management of children with cerebral palsy: indications and outcome. Eur J Neurol 4(Suppl. 2):S15-S22.

Boyd RN, Pliatsios V, Wolfe R, Graham HK (2001). Use of objective clinical measures in predicting response to botulinum toxin A in children with cerebral palsy. Dev Med Child Neurol in press.
Boyd RN, Starr R, Wolfe R, Graham HK (2000). Biomechanical transformation of the gastroc-soleus muscle with botulinum toxin A in children with cerebral Palsy. Dev Med Child Neurol 42:32-41.

Calderon-Gonzalez R, Calderon-Sepulveda R, Rincon-Reyes M, Garcia-Ramirez J, Mino-Arango A (1994). Botulinum toxin A in management of cerebral palsy. Pediatric Neurol 10:284-288.

Corry I, Cosgrove A, Duffy C, McNeil S, Taylor T, Graham $\mathrm{H}$ (1998). Botulinum toxin A compared with stretching casts in the treatment of spastic equinus, a randomised prospective trial. J Pediat Orthoped 18:304-311.

Corry IS, Cosgrove AP, Graham HK (1999). Botulinum toxin A in hamstrings spasticity. Gait Posture 10:206-210.

Cosgrove AP, Corry IS, Graham HK (1994). Botulinum toxin in the management of the lower limb in cerebral palsy. Dev Med Child Neurol 36:386-396.

Delp SL, Arnold AS, Speers RA, Moore CA (1996). Hamstrings and psoas lengths during normal and crouch gait: implications for muscle-tendon surgery. J Orthopaedic Res 14:144-151.

Desloovere K, Molenaers G, Eyssen M (1998). The three dimensional kinematics, kinetics and EMG pattern of the walking gait cycle of children aged between three and six years. Gait Posture 8:76.

Desloovere K, Molenaers G, Jonkers I, Van Deun S, Nijs J (2000). The effect of combined botulinum toxin injections and serial casting on gait disorders in cerebral palsy. Gait Posture 12:57.

Flett PJ, Stern LM, Waddy H, Connell TM, Seeger JD, Gibson SK (1999). Botulinum toxin A versus fixed cast stretching for dynamic calf tightness in cerebral palsy. $J$ Paediatric Child Health 35:71-77.

Frossberg H, Tedroff KB (1997). Botulinum toxin treatment in cerebral palsy: intervention with poor evaluation? Dev Med Child Neurol 39:635-640.

Gage JR (1991).Gait Analysis in Cerebral Palsy. London: Mac Keith Press.

Graham HK, Aoki KR, Autti-Rmo I et al. (2000). Recommendations for the use of botulinum toxin type $\mathrm{A}$ in the management of cerebral palsy. Gait Posture 11:67-79.

Hesse S, Reiter F, Konrad M, Jahnke MT (1998). Botulinum toxin type A and short-term electrical stimulation in the treatment of upper limb flexor spasticity after stroke: a randomized, double-blind, placebo controlled trial. Clin Rehahabilitation 12:381-388.

Inman VT, Ralston HJ, Todd F (1991). Human Walking. Baltimore: Williams \& Wilkins.

Koman LA, Mooney JF, Smith BP, Goodman A, Mulvaney T (1994). Management of spasticity in cerebral palsy with botulinum-A toxin: report of a preliminary, randomized, double-blind trial. J Pediat Orthoped 14:299-303.

Molenaers G, Desloovere K, Eyssen M, De Cat J, Jonkers I, De Cock P (1999a). Botulinum toxin type A treatment of cerebral palsy: an integrated approach. Eur J Neurol 6:S51S57.

Molenaers G, Desloovere KI, Jonkers F, Glorieux De Cock P (1999b). The effect of multilevel botulinum toxin type A treatment combined with short leg casting and othotic management on the gait of CP children. Gait Posture 10:74.

Molenaers G, Eyssen M, Desloovere K, Jonkers I, De Cock P (1999c). A multilevel approach to botulinum toxin typa A treatment of the (ilio) psoas in spasticity in cerebral palsy. Eur J Neurol 6:S59-S62. 
Motta F (1997). The effect of Botulinum-A toxin treatment in children with spastic cerebral palsy: kinematic and kinetic aspects of ankle and knee joints. Gait Posture 6:275.

Perry J (1992). Gait Analysis: normal and pathological function. Thorofare, New Jersey; SLACK Incorporated.

Suliman IA, Lindgren JU, Gillberg PG, Diab KM, Adem A (1997). Effect of immobilization on skeletal muscle nicotinic cholinergic receptors in the rat. Neuroreport 8: 2821-2824.

Sutherland DH, Kaufman K, Wyatt M, Chambers H (1996). Injection of botulinum A toxin into the gastrocnemius muscle of patients with cerebral palsy: a 3-dimensional motion analysis study. Gait Posture 4:269-279.

Wenger DR, Rang M (1993).The Art and Practice of Children's Orthopaedics. New York; Raven Press.

Winter DA (1987).The Biomechanics of Motor Control of Human Walking. Ontario: University of Waterlo Press.

Wissel J, Heinen F, Schenkel A (1999). Botulinum toxine A in the management of spastic gait disorders in children and young adults with cerebral palsy: a randomised double blind study of 'high dose' versus 'low dose' treatment. Neuropediatrics 30:120-124. 